\title{
Gas Sensing using Material and Structural Slow Light Systems
}

\author{
Luc Thévenaz ${ }^{1}$, Isabelle Dicaire ${ }^{1}$, Sanghoon Chin ${ }^{1}$, Alfredo De Rossi ${ }^{2}$ \\ ${ }^{1}$ Ecole Polytechnique Federale de Lausanne, Institute of Electrical Engineering, SCI-STI-LT Station 11, 1015 Lausanne, Switzerland. \\ ${ }^{2}$ Thales Research and Technology, Route Départementale 128, 91767 Palaiseau Cedex, France. \\ Authore-mail address: Luc.Thevenaz@EPFL.CH
}

\begin{abstract}
Slowing light is expected to enhance light-matter interaction, leading to more compact sensing devices. By simple tests we show that the reality is more complex and the only relevant quantity is the wave electric field.

OCIS codes: Spectroscopy: 300.1030 Absorption, Remote sensing and sensors: 280.4788 Optical sensing and sensors, Nonlinear optics: 190.5890 Scattering, stimulated.
\end{abstract}

\section{Introduction}

Photonic crystal $(\mathrm{PhC})$ waveguides are attractive evanescent field devices for the detection of hazardous gases and analytes in aqueous environment. They require small sample volumes and short response times due to their ultracompact dimensions. Refractive index sensing was recently demonstrated in $\mathrm{PhC}$ waveguides using $\mathrm{PhC}$ optical cavities with air slots embedded in the defect region [1-3]. While this technique allows for very sensitive measurements of refractive index changes it cannot specifically identify the chemical species under study. In this case infrared (IR) absorption spectroscopy is a powerful and highly selective technique that can identify molecular species from their individual spectroscopic fingerprint. The Beer-Lambert law describes the intensity of a light signal decaying through an absorbing medium:

$$
I=I_{o} \exp (-\Gamma \alpha L)
$$

where $I_{0}$ is incident light intensity, $\alpha$ is the highly-selective wavelength-dependent absorption coefficient, $L$ is the optical path length, and $\Gamma$ is the absorption enhancement factor. Since the optical path length is notably small in PhC waveguides it was previously suggested to use slow light propagation to locally enhance light-matter interactions, thus enhancing the absorption sensitivity [4]. Gas sensing measurements of carbon dioxide gas and methane gas were recently performed in dispersion-engineered bulk $\mathrm{PhCs}$ and $\mathrm{PhC}$ waveguides, respectively [5-6]. Experimental enhancement factors $\Gamma$ of 2-3 were reported in [5] for group indices of $n_{g} \sim 2$ while higher absorption sensitivity was reported in [6] but the associated enhancement factors were not experimentally obtained.

High-resolution IR absorption spectroscopy of acetylene gas is here demonstrated in dispersion-engineered GaInP PhC waveguides under slow light propagation. Individual absorption profiles are obtained for both $\mathrm{TE}$ and TM polarizations for group indices ranging from 1.5 to 6.7. Experimental enhancement factors are obtained by measuring the corresponding absorption coefficients under free-space propagation for exactly the same path length. The gas sensing measurements are confirmed by numerical simulations of molecular absorption from the evanescent field of $\mathrm{PhC}$ waveguides in the presence of wavelength-dependent dispersion.

\section{Description of the experiment}

The PhC waveguide samples used in this study consist of an 180nm-thick GaInP slab with a triangular lattice of air holes having a lattice constant of $486 \mathrm{~nm}$ and a defect line. To modify their structural dispersion properties, the size of the first row of holes was increased from $204 \mathrm{~nm}$ to $233 \mathrm{~nm}$ in the first sample and from $224 \mathrm{~nm}$ to $253 \mathrm{~nm}$ in the second sample. The $1.5 \mathrm{~mm}$-long waveguides include mode adapters on both end facets to gradually build the slowlight mode and to reduce Fabry-Perot oscillations due to the strong impedance mismatch between incident modes (free-space or optical fiber modes) and waveguides modes [7].

Molecular spectroscopy was performed in $\mathrm{PhC}$ waveguides using the following experimental procedure. The optical coupling setup comprising two micropositioning stages and a moving sample holder was placed inside a gas chamber having optical fibre feedthrough for remote monitoring. The polarization state incident on the samples was set to TE and TM independently using a linear polarizer providing a fixed arbitrary polarization followed by a polarization controller. The TE polarization state was verified by observing a cut-off in the transmission spectrum of a $\mathrm{PhC}$ waveguide using an optical spectrum analyzer (OSA) and a broadband source emitting in the range 1520$1570 \mathrm{~nm}$. TM polarization was set by observing no spectral cut-off on the OSA, indicating that light no longer experienced multiple reflections by the periodic $\mathrm{PhC}$ structure. 
The gas chamber was then hermetically closed and air was evacuated using a vacuum pump without causing any significant change in transmitted intensity $( \pm 1 \mathrm{~dB})$. The gas chamber was filled with 50 torrs (67 mbars) of acetylene gas; this value was chosen to maximize absorption coefficients while keeping narrow linewidths. To obtain highresolution absorption profiles the injection current of a distributed-feedback (DFB) laser diode operating at $1534.1 \mathrm{~nm}$ was modulated as a ramp signal to sweep the DFB laser line across the P15 absorption line. Group indices were measured using an optical frequency domain interferometric technique comprising a tunable laser source and a Mach-Zehnder interferometer with the $\mathrm{PhC}$ waveguide sample placed in one arm and followed by digital filtering.

\section{Results}

The experimental acetylene absorption lines obtained using dispersion-engineered $\mathrm{PhC}$ waveguides are presented in Fig. 1 for both TE and TM polarizations (blue and green solid curves, color online) and for two different waveguides with different $n_{g}$ values. The dashed curve represents absorption under free-space propagation for exactly the same path length ( $L=1.5 \mathrm{~mm}$, Gaussian fit of dotted profile).
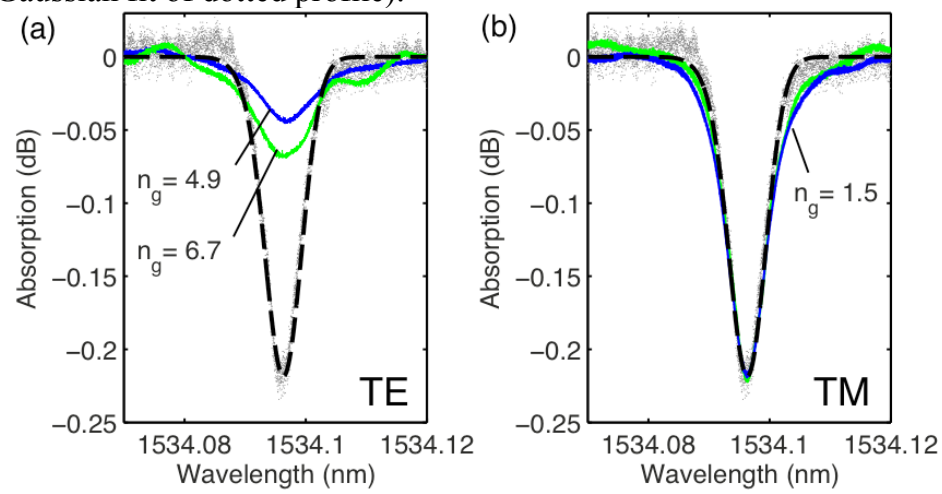

Fig. 1. (a) Acetylene P15 absorption line for the TE polarization for $n_{g}=4.9$ (blue) and $n_{g}=6.7$ (green). Experimental free-space absorption (dotted profile) and its Gaussian fit (dashed curve) are also shown. (b) Same for TM polarization, $n_{g}=1.5$ for the two PhC waveguide samples.

As illustrated in Fig. 1, the experimental absorption efficiency is greater for the TM polarization with $n_{g}=1.5$ than for the TE one for which $n_{g}=[4.9 ; 6.7]$; this is a very surprising result considering the expected enhancement of molecular absorption due to slow light propagation that occurs only for the TE mode. This is due to the fact that for TE polarization most of the electric field is located inside the high-index dielectric slab while for TM polarization the electric field is enhanced in the lower-index material. Boundary conditions at material interfaces state that for an electric field perpendicular to the high-index dielectric slab (i.e. TM pol.) the electric displacement field $D=\varepsilon E$ must be continuous at its interface, resulting in a relative evanescent electric field enhancement of $\varepsilon_{\text {slab }} / \varepsilon_{0}$ for TM polarization and a continuous weak evanescent field for TE polarization. Such low electric field intensities for TEpolarized light can be circumvented by adding a narrow air slot in the defect region of a $\mathrm{PhC}$ waveguide, thereby causing a large field discontinuity for the TE polarization and electric field enhancement in the air slot [8].

To experimentally compare the dependence of absorption efficiencies on group indices several conditions must be satisfied. One important requirement is that the measured profiles should have the same linewidths since the absorption efficiency will depend on the absorption linewidth for any Lorentzian or Gaussian profile. The intensity of the electric field $|E|^{2}$ located within the lower index material should also be identical for all measurements. Table 1 presents the characteristics of the experimental absorption profiles for both TE and TM polarizations.

Table 1. Characteristics of experimental and simulated absorption coefficients

\begin{tabular}{|c|c|c|c|c|c|}
\hline Line & $\begin{array}{c}\text { Group } \\
\text { Index }\end{array}$ & $\begin{array}{c}\text { Linewidth } \\
{[\mathbf{G H z}]}\end{array}$ & $\begin{array}{c}\text { Experimental } \\
\text { Absorption [dB] }\end{array}$ & $\begin{array}{c}\text { Experimental } \\
\text { Enhancement }\end{array}$ & $\begin{array}{c}\text { Simulated } \\
\text { Enhancement }\end{array}$ \\
\hline \hline TE & 4.9 & $1.4 \pm 0.1$ & $0.044 \pm 0.001$ & $0.20 \pm 0.01$ & 0.19 \\
TE & 6.7 & $1.5 \pm 0.3$ & $0.068 \pm 0.001$ & $0.31 \pm 0.02$ & 0.25 \\
TM & 1.5 & $0.94 \pm 0.01$ & $0.219 \pm 0.001$ & $1.00 \pm 0.05$ & 1.12 \\
Free space & 1.0 & $0.87 \pm 0.05$ & $0.22 \pm 0.01$ & $1.00 \pm 0.05$ & 1.00 \\
\hline
\end{tabular}


Since TE-polarized absorption profiles have approximately the same linewidth and mode field distribution the absorption efficiencies can be directly compared to group indices. One finds that $A\left(n_{g}=4.9\right) / A\left(n_{g}=6.7\right) \cong 4.9 / 6.7$, i.e. the absorption coefficient is approximately linearly proportional to $n_{g}$ when all other relevant quantities are equal.

\section{Analysis and discussion}

To confirm this linear dependence time-domain simulations of molecular absorption were realized and the obtained electromagnetic field distributions and dispersion relation were then used to calculate absorption enhancement factors. Theoretically the absorption coefficient is given by the coupling between the electric field of the optical wave and the electric dipole induced by the bound orbiting electrons of molecules, divided by the Poynting vector averaged over the waveguide cross section:

$$
\alpha=-\frac{d I}{I \cdot d x} \approx \frac{\int_{\text {gas }} \mathfrak{I}[\varepsilon]|E|^{2} d V / 2}{\mathfrak{R}\left[\int_{A} E \times H^{*} d A\right]},
$$

where $H$ is the magnetic field, $\varepsilon$ is the complex electric permittivity, and the functions $\mathfrak{R}$ and $\mathfrak{I}$ denote the real and imaginary parts of a complex number. Since the Poynting vector is proportional to the velocity of the electromagnetic energy $v_{E M}$, Eq. (2) reduces to the following expression (where $f_{E}$ is the overlap fraction of the electric field with absorbers):

$$
\alpha \propto n_{g} \cdot f_{E},
$$

which is valid in photonic crystal waveguides since $v_{E M}=v_{g}$ in such structures [9]. Figure 2 presents the theoretical absorption coefficient normalized by that obtained in free-space propagation for both TE and TM polarizations.
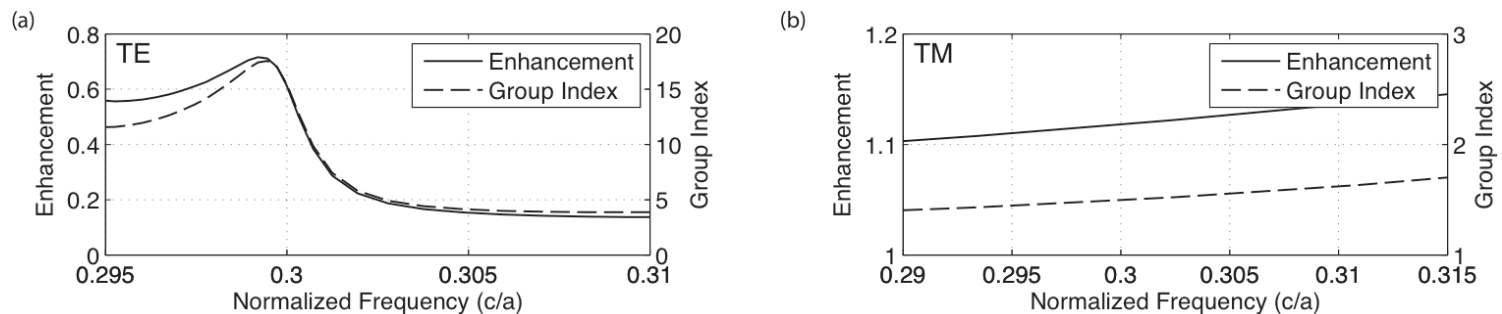

Fig. 2. (a) Simulated enhancement factors (solid line) and group indices (dashed line) as a function of the normalized frequency for TE-polarized light. (b) Same for TM-polarized light.

As expected, there is no absorption enhancement for the TE polarization due to low electric field overlap fractions, while strong absorption is obtained for TM polarization due to the electric field enhancement resulting from its discontinuity at the dielectric interface. Experimental and theoretical absorption coefficients are compared in Table 1 and agree within 20 percent, confirming the linear dependence of molecular absorption on the group index. These results indicate that the distribution of the electric field intensity is fundamental in gas-sensing measurements and must not be confused with the optical intensity distribution: they are two entirely different quantities in PhC waveguides. Practically, it turns out that enhancing the electric field intensity overlapping the absorbers shows a more decisive impact on the absorption enhancement than searching for extreme group index.

\section{References}

[1] Di Falco, A., O’Faolain, L., and Krauss, T. F., "Chemical sensing in slotted photonic crystal heterostructure cavities", Appl. Phys. Lett. 94, 063503 (2009).

[2] Jagerska, J., Zhang, H., Diao, Z., Thomas, N. L., and Houdré, R., "Refractive index sensing with an air-slot photonic crystal nanocavity", Opt. Lett. 35, 2523-2525 (2010).

[3] Wang, B., Dündar, M. A., Nötzel, R., Karouta, F., He, S., and van der Heijden, R. W., "Photonic crystal slot nanobeam slow light waveguides for refractive index sensing", Appl. Phys. Lett. 97, 151105 (2010).

[4] Mortensen, N. A. and Xiao, S., "Slow-light enhancement of Beer-Lambert-Bouguer absorption", Appl. Phys. Lett. 90, 141108 (2007).

[5] Pergande, D., Geppert, T. M., Rhein, A. v., Schweizer, S. L., Wehrspohn, R. B., Moretton, S., and Lambrecht, A., "Miniature infrared gas sensors using photonic crystals", Journal of Appl. Phys. 109, 083117 (2011).

[6] Lai, W. C., Chakravarty, S., Wang, X., Lin, C., and Chen, R. T.,"On-chip methane sensing by near-IR absorption signatures in a photonic crystal slot waveguide", Opt. Lett. 36, 984-986 (2011).

[7] Tran, Q. V., Combrié, S., Colman, P., and Rossi, A. D., "Photonic crystal membrane waveguides with low insertion losses", Appl. Phys. Lett. 95, 061105 (2009).

[8] Almeida, Q. Xu, C.A. Barrios, and M. Lipson, “Guiding and confining light in void nanostructure,” Opt. Lett. 29, 1209-1211 (2004).

[9] Sakoda, K., "Optical Properties of Photonic Crystals”, Springer, Heidelberg, $2^{\text {nd }}$ Edition (2005). 